\title{
Lung Stereotactic Body Radiotherapy Using an Abdominal Compression System, “Air-Bag System"
}

\author{
Hideharu Miura*, Ryoong-Jin Oh, Norihisa Masai, Hiroya Shiomi, Kouichi Yamada, \\ Toshihiko Inoue
}

Miyakojima IGRT Clinic, 1-16-22 Miyakojima Hondori, Miyakojima-ku, Osaka, Japan

Email: ${ }^{*}$ hide-miura@osaka-igrt.or.jp

Received 26 March 2014; revised 16 April 2014; accepted 13 May 2014

Copyright (C 2014 by authors and Scientific Research Publishing Inc.

This work is licensed under the Creative Commons Attribution International License (CC BY).

http://creativecommons.org/licenses/by/4.0/

(c) (i) Open Access

\begin{abstract}
We investigated respiratory tumor motion in lung stereotactic body radiotherapy (SBRT) with use of the "Air-Bag System". 114 patients underwent four-dimensional (4D) computed tomography (CT) from October 2010 to April 2012. Gross tumor volume (GTV) was $8.1 \pm 11.0$ cc (range 0.3 $77.5 \mathrm{cc}$ ). The tumor site was the upper and middle lobes in 62 cases, and lower lobe in 52 cases. The Air-Bag System ${ }^{\mathrm{TM}}$ consists of an inelastic air bag connected to a second smaller elastic air bag. The inelastic air bag is placed between the patient's body surface and a HipFix and is secured by pressure adjustment via the elastic air bag. To assess respiratory tumor motion, the centroid of the tumor position is measured in the left-right, anterior-posterior, and caudal-cranial directions using the iPlan RT Dose ${ }^{\mathrm{TM}}$ treatment planning system. Respiratory tumor motion vector for patients with upper/middle and lower lobe tumors was $3.0 \pm 2.2 \mathrm{~mm}$ (range, $0.4-11.7 \mathrm{~mm}$ ) and $6.5 \pm$ $4.6 \mathrm{~mm}$ (range, $0.4-22.0 \mathrm{~mm}$ ) respectively, with this difference being significant $(p<0.05)$. Mean respiratory tumor motion for all patients was $0.9 \pm 0.6 \mathrm{~mm}$ (range, $0.1-3.6 \mathrm{~mm}$ ) in the left-right direction, $1.5 \pm 1.1 \mathrm{~mm}$ (range, $0.1-5.7 \mathrm{~mm}$ ) in the anterior-posterior direction, $4.1 \pm 4.0 \mathrm{~mm}$ (range, $0.1-21.4 \mathrm{~mm}$ ) in the caudal-cranial direction, and $4.7 \pm 4.0 \mathrm{~mm}$ (range, $0.4-22.0 \mathrm{~mm}$ ) overall. The Air-Bag System is expected to be provided an effective reduction in the motion of lung tumors.
\end{abstract}

\section{Keywords}

Stereotactic Body Radiotherapy, Lung Cancer, Tumor Motion, Abdominal Compression, Air-Bag System

\footnotetext{
"Corresponding author.
}

How to cite this paper: Miura, H., et al. (2014) Lung Stereotactic Body Radiotherapy Using an Abdominal Compression System, "Air-Bag System". International Journal of Medical Physics, Clinical Engineering and Radiation Oncology, 3, 98-106. 


\section{Introduction}

Intrafraction motion is an important issue in the era of image-guided radiotherapy. Intrafraction motion can be caused by the respiratory, skeletal muscular, cardiac, and gastrointestinal systems [1]. Lung tumor motion is related to breathing, which produces the greatest movement in the caudal-cranial direction [1]-[4]. Daily patient set-up should take account of internal variability in tumor position independently of bony anatomy or the external stereotactic system [5]. To achieve this, an internal margin should be added around the clinical target volume (CTV) to account for respiratory tumor motion [6]. However, this margin results in an increased dose to the normal tissue, which increases the risk of normal tissue toxicity and limits dose-escalation, and in turn leads to a higher probability of radiation pneumonitis [7]. Methods to reduce the margin include delivery techniques such as respiratory gating, active breathing control, real-time tracking, and voluntary breath holding [8], but these require a longer time for beam delivery and are more difficult for old patients, who must maintain normal breathing over the delivery time. We consider that respiratory tumor motion is best controlled under free breathing using an abdominal compression system to suppress respiratory tumor motion.

Abdominal compression has been widely used to minimize respiratory-associated tumor motion in lung stereotactic body radiotherapy (SBRT). One such system is the Stereotactic Body Frame (Elekta, Crawley, UK), which can be used in combination with an abdominal pressure board [9]. However, this system is not comfortable for the patient. We have therefore used the Air-Bag System ${ }^{\mathrm{TM}}$ (Niigata Kiden Ltd., Niigata, Japan), which uses abdominal compression to suppress respiratory tumor motion.

The purpose of this study was to investigate respiratory tumor motion using the Air-Bag System.

\section{Materials and Methods}

\subsection{Patient Characteristics}

114 patients with localized lung tumors suitable for SBRT were enrolled between October 2010 and April 2012. Patient characteristics are shown in Table 1. Gross tumor volume (GTV) and patient age did not significantly differ between patients with tumors in the upper/middle and lower lobes.

\subsection{Principle of the Air-Bag System}

The Air-Bag System consists of an inelastic air bag connected to a second smaller elastic air bag, air flow sensor and air supply pump (Figure 1). The length and width of the inelastic air bag are 250 and 460 mm, respectively. Figure 2 shows the process of acquiring the respiratory wave using the Air-Bag System. The inelastic air bag is placed between the patient's body and the stereotactic fixation frame. The air bag is then inflated using an air supply pump. The two bags are interconnected. An air flow meter device located between the two air bags measures "air flow" back and forth between the two bags. These data are processed using a computer and translated to provide a highly accurate respiratory motion curve. This curve is displayed as a swinging decoy marker on the computer screen to allow the infrared camera of the Real-Time Position Management System ${ }^{\mathrm{TM}}$ (RPM) (Varian Medical Systems, Palo Alto, CA) to capture the breathing pattern (Figure 3). The Air-Bag System can be easily and immediately connected in a plug-and-play structure, and can be interfaced on use with the RPM system's infrared camera. Thanks to this, the Air-Bag System does not require specific patient data, treatment data, or input of any particular information from other devices, and can be easily integrated with the existing

Table 1. Patient characteristics.

\begin{tabular}{|c|c|c|c|}
\hline & & & $\mathrm{N}=114$ \\
\hline & Upper and middle lobes & Lower lobe & p value \\
\hline Target location (n) & 62 & 52 & $\mathrm{n} / \mathrm{a}^{* *}$ \\
\hline Gender (n) men & 42 & 38 & $\mathrm{n} / \mathrm{a}$ \\
\hline Women & 20 & 14 & $\mathrm{n} / \mathrm{a}$ \\
\hline Age (y) & $71 \pm 13(34-89)$ & $73 \pm 8(48-88)$ & 0.122 \\
\hline Gross tumor volume (cc) & $7.1 \pm 8.4(0.3-38.3)$ & $10.4 \pm 14.0(0.3-77.5)$ & 0.139 \\
\hline
\end{tabular}

Gross tumor volume (GTV) and age did not significantly differ between upper/middle and lower lobes. *Student unpaired t-test; ${ }^{* *}$ not applicable. 


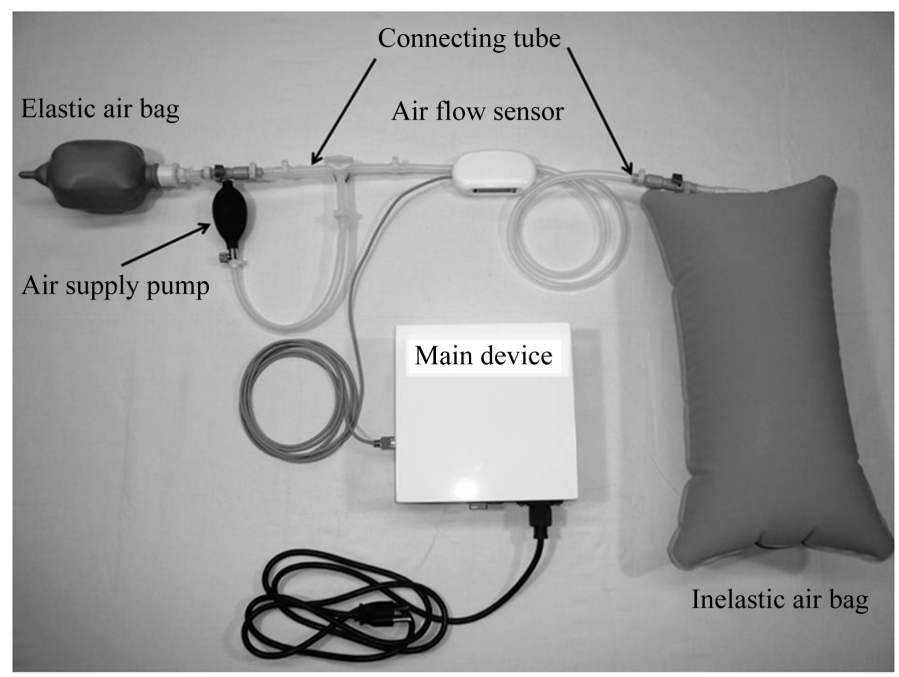

Figure 1. Air-Bag System ${ }^{\mathrm{TM}}$.

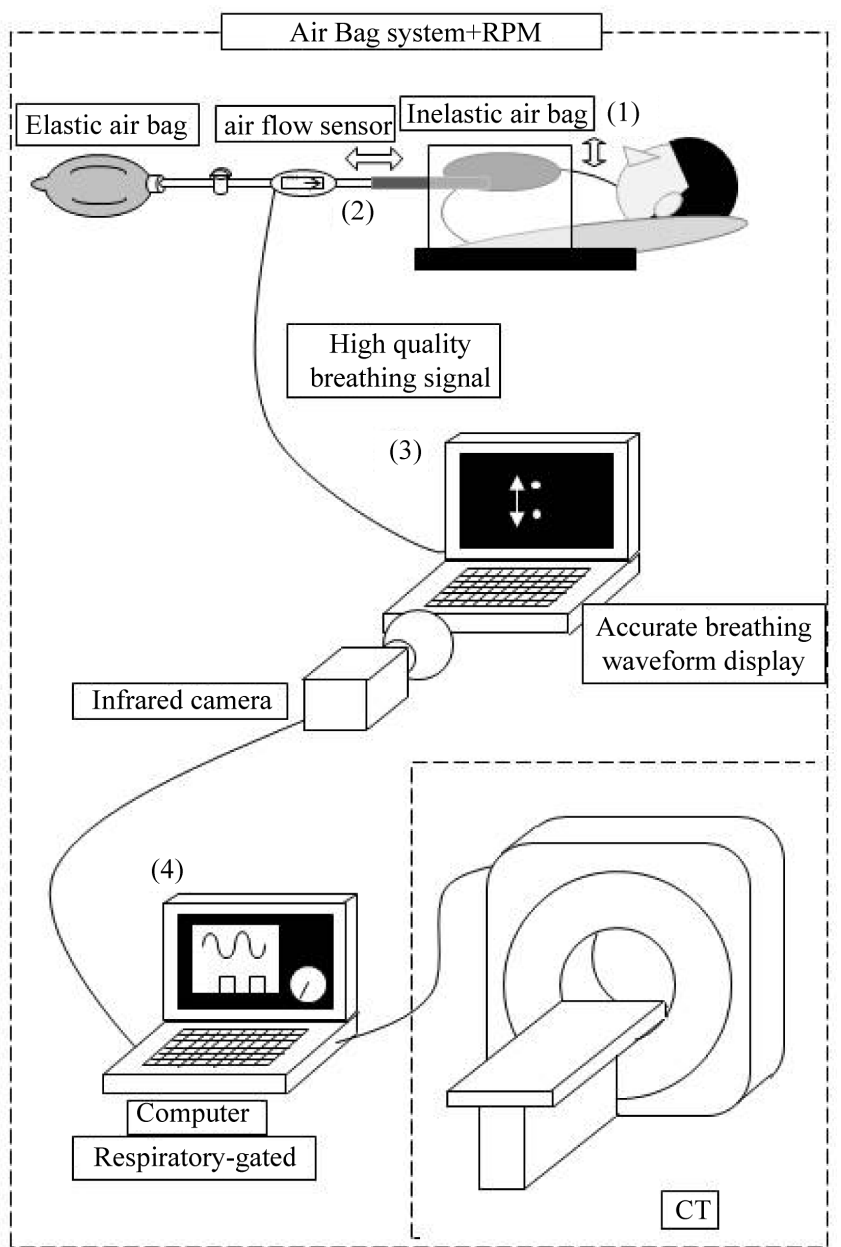

Figure 2. Schematic diagram of the respiratory wave acquired with AirBag system. (1) Air flow change in synchronization with abdominal motion. (2) Air flow is translated into digital data. (3) Digital data is displayed as a swinging decoy marker on the computer screen. (4) The decoy marker can be detected by the infrared camera of the RPM system. 

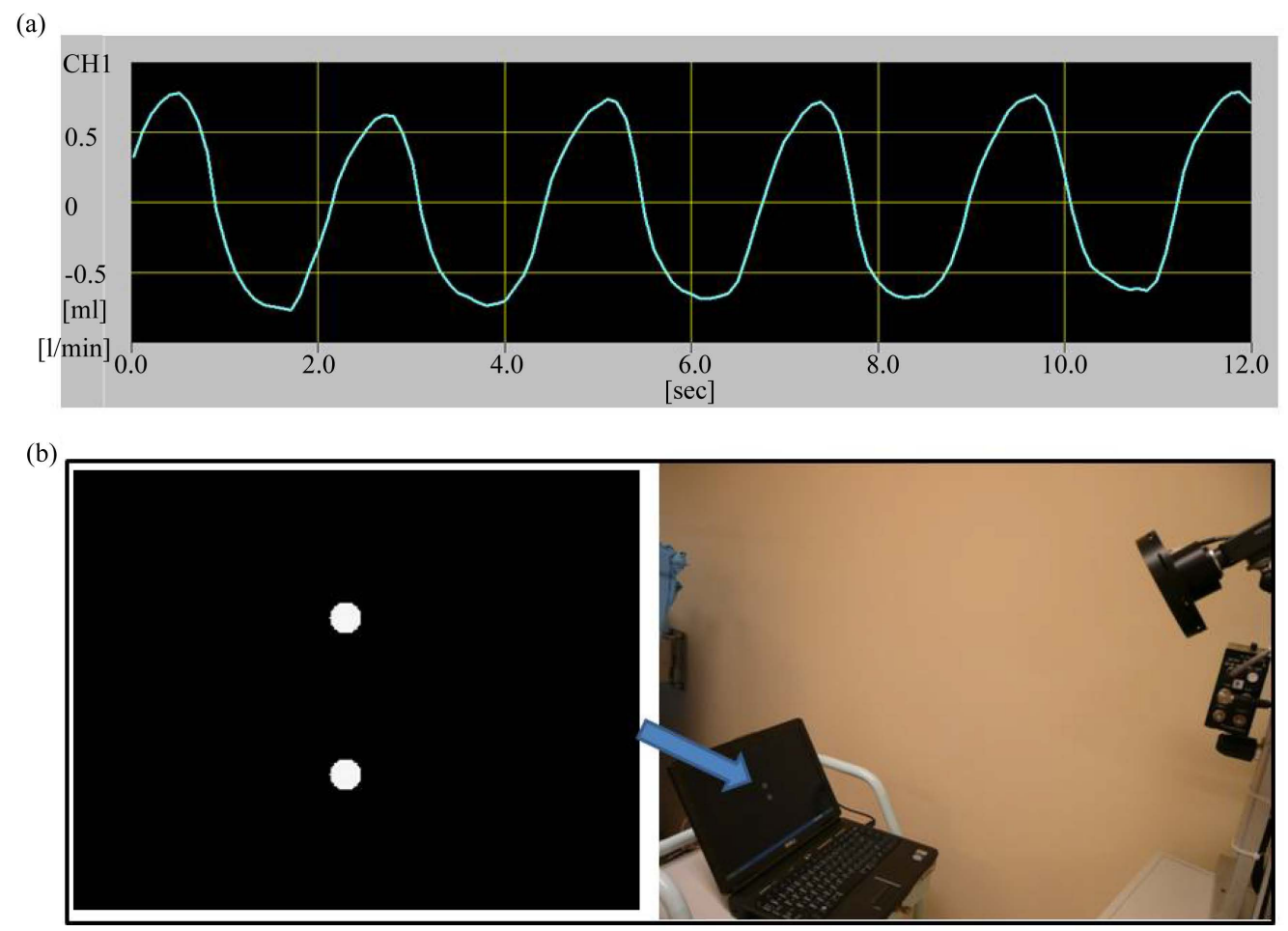

Figure 3. (a) Amplifying the respiratory waveform allows the phase of expiratory tumor motion to be easily observed. (b) The respiratory wave can be displayed as a decoy marker.

RPM System infrared camera. Further, four-dimensional (4D)-Computed Tomography (CT) can be performed using the existing RPM system with the swinging decoy.

\subsection{Treatment Procedure}

The patient is placed in the supine position on an evacuated Vac-Lok ${ }^{\mathrm{TM}}$ immobilization device (CIVCO Medical Solutions, South Kalona, Iowa, USA) with the arms above the head (Figure 4(a)). The Vac-Lok ${ }^{\mathrm{TM}}$ is evacuated using a vacuum pump so that it molds to the patient's posterior body surface. HipFix (CIVCO Medical Solutions, USA) is attached to the sides of the Vac-Lok ${ }^{\mathrm{TM}}$ to mold the patient's body surface to the level of the abdomen or thorax. The inelastic air bag is placed between the patient's body surface and the HipFix ${ }^{\mathrm{TM}}$ and secured by pressure adjustment via the elastic air bag (Figure 4(b), Figure 4(c) and Figure 5). The air bag can be inflated by injection of 2000 cc of air using an air supply pump. If a patient experiences excessive air pressure, the volume of air can be reduced. The flow of air displayed on the sensor screen reflects the breathing of the patient, and this information is then used as output towards the RPM device.

As a routine procedure in the planning of stereotactic radiotherapy, a whole chest 3D-CT series was obtained under free breathing using the Air-Bag System, with acquisition on a 4-slice Brightspeed QX/i ${ }^{\mathrm{TM}}$ scanner (GE Medical Systems, Waukesha, WI). Tumor shape, volume, and position at different phases of the breathing cycle were more accurately determined using 4D-CT. The CT images had a slice thickness of $2.5 \mathrm{~mm}$ with a gantry rotation time of 1.0 second. Respective images were tagged with the corresponding phase of the respiratory cycle and then sent to the Advantage Workstation ${ }^{\mathrm{TM}}$ (GE Medical Systems) using the Advantage 4D-CT software. The $4 \mathrm{D}$ datasets were categorized into four phases of the respiratory cycle of $0 \%, 25 \%, 50 \%$, and $75 \%$, with $0 \%$ representing maximum inspiration.

Image registration was fused on the bony anatomy using the iPlan RT image ver 4.1.1 ${ }^{\mathrm{TM}}$ treatment planning system (Brainlab, Munich, Germany). The GTV was contoured by a single physician on each axial slice. The window width and level used to define the tumor target were $1200 \mathrm{HU}$ and $-400 \mathrm{HU}$, respectively. The CTV was defined as the same volume as the GTV in almost all cases. The internal target volume (ITV) was the sum of the GTV positions in all respiratory phases. 

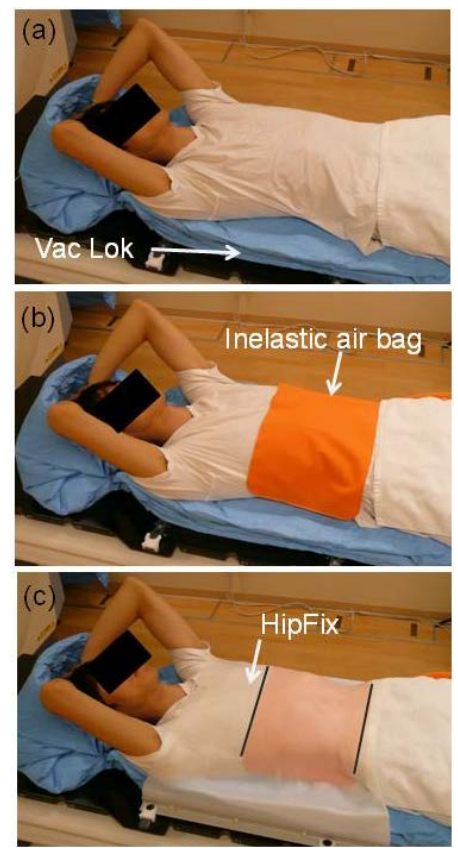

Figure 4. Use of the Air-bag system. (a) The patient lies down in a supine position. (b) The inelastic air bag is placed in the same position as the patient abdomen. (c) The inelastic air bag is placed between patient's body surface and the HipFix device, which is fixed on the couch.

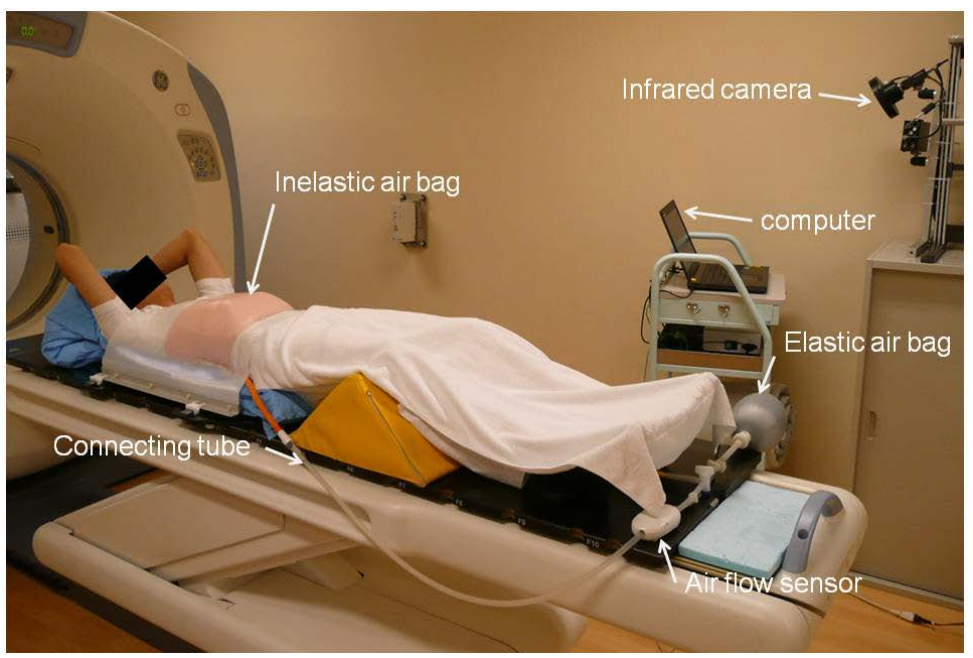

Figure 5. Patient lies down in a supine position with the Air-Bag System.

The planning target volume (PTV) was generated by adding a uniform margin of $8 \mathrm{~mm}$ to the ITV to account for set-up uncertainties and mechanical inaccuracy. The treatment fields were conformed around the PTV. A leaf margin of $2 \mathrm{~mm}$ was added to the PTV, and the isocenter was positioned in the center of the PTV. Treatment plan characteristics have been described previously [10].

\subsection{Data Analysis}

Respiratory tumor motion was evaluated using the 4D-CT images, and measured from the displacement of the centroid of the GTV in the different phases. The geometric centroid of each GTV volume was determined in iplan 
RT Dose. Respiratory tumor motion was classified into three different directions, with the X (left-right), Y (anterior-posterior) and Z (caudal-cranial) positions determined for each phase. Motion was then defined as the absolute difference between the maximum coordinate and minimum coordinate of each phase and combined in quadrature to obtain the magnitude of the three-dimensional (3D) vector, calculated by:

$$
\sqrt{\mathrm{X}^{2}+\mathrm{Y}^{2}+\mathrm{Z}^{2}}
$$

Differences in the upper/middle and lower lobes were analyzed using the Student unpaired t-test, with p-values less than 0.05 considered statistically significant. The analyzed data were displayed as the mean \pm standard deviation with ranges in parentheses among the 114 clinical plans.

\section{Results}

Figure 6 shows plots of respiratory tumor motion of the upper/middle and lower lobes. Figure 7 shows the mean respiratory tumor motion of the upper/middle and lower lobes. Respiratory tumor motion in patients with tumors in the upper/middle and lower lobes was greatest in the caudal-cranial direction, at $2.3 \pm 2.2 \mathrm{~mm}$ (range, $0.1-11.3 \mathrm{~mm}$ ) and $6.3 \pm 4.6 \mathrm{~mm}$ (range, $0.3-21.4 \mathrm{~mm})(\mathrm{p}<0.05)$, respectively. Respiratory tumor motion in the left-right direction with upper/middle and lower lobe tumors was $0.9 \pm 0.7 \mathrm{~mm}$ (range, 0.1 - $3.6 \mathrm{~mm}$ ) and 0.9 $\pm 0.6 \mathrm{~mm}$ (range, $0.1-2.9 \mathrm{~mm})(\mathrm{p}=0.74)$, respectively, while that in the anterior-posterior direction was $1.5 \pm$ $1.0 \mathrm{~mm}$ (range, $0.1-5.7 \mathrm{~mm}$ ) and $1.5 \pm 1.2 \mathrm{~mm}$ (range, $0.1-5.1 \mathrm{~mm})(\mathrm{p}=0.39$ ), respectively. Respiratory tumor motion vector for patients with upper/middle and lower lobe tumors was $3.0 \pm 2.2 \mathrm{~mm}$ (range, $0.4-11.7$ $\mathrm{mm}$ ) and $6.5 \pm 4.6 \mathrm{~mm}$ (range, $0.4-22.0 \mathrm{~mm}$ ), respectively, with this difference being significant $(\mathrm{p}<0.05$ ). Mean respiratory tumor motion for all patients was $0.9 \pm 0.6 \mathrm{~mm}$ (range, $0.1-3.6 \mathrm{~mm}$ ) in the left-right direction, $1.5 \pm 1.1 \mathrm{~mm}$ (range, $0.1-5.7 \mathrm{~mm}$ ) in the anterior-posterior direction, $4.1 \pm 4.0 \mathrm{~mm}$ (range, $0.1-21.4 \mathrm{~mm}$ ) in the caudal-cranial direction, and $4.7 \pm 4.0 \mathrm{~mm}$ (range, $0.4-22.0 \mathrm{~mm}$ ) overall. Respiratory tumor motion of more than $20 \mathrm{~mm}$ was seen in one patient, who had a lower lobe tumor.

\section{Discussions}

This study demonstrates the benefits of the Air-Bag System in respiratory tumor motion using abdominal compression to produce a highly efficient decrease in breathing-induced tumor motion. Our results indicate that average respiratory tumor motion with the Air-Bag System was 3.0 and $6.5 \mathrm{~mm}$ for upper/middle and lower lesions, respectively. Respiratory tumor motion range in the upper/middle lobe along the cranio-caudal direction was significantly smaller than that in the lower lobe. Respiratory tumor motion of more than $20 \mathrm{~mm}$ was seen in

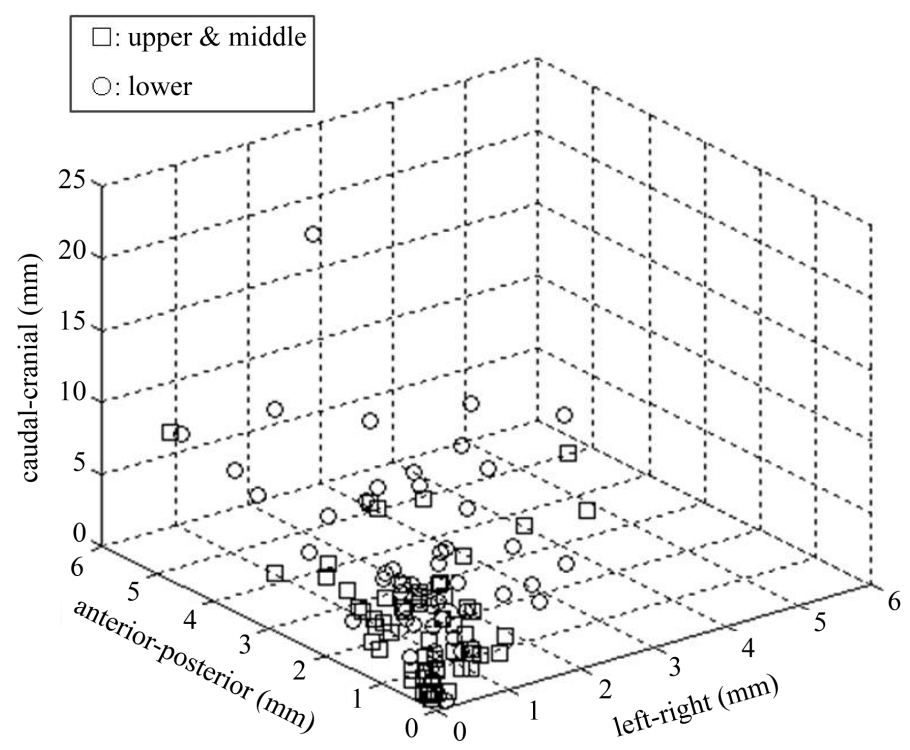

Figure 6. Plots of respiratory tumor motion of the upper/middle (square) and lower (circle) lobes using the Air-Bag System in 114 patients. 


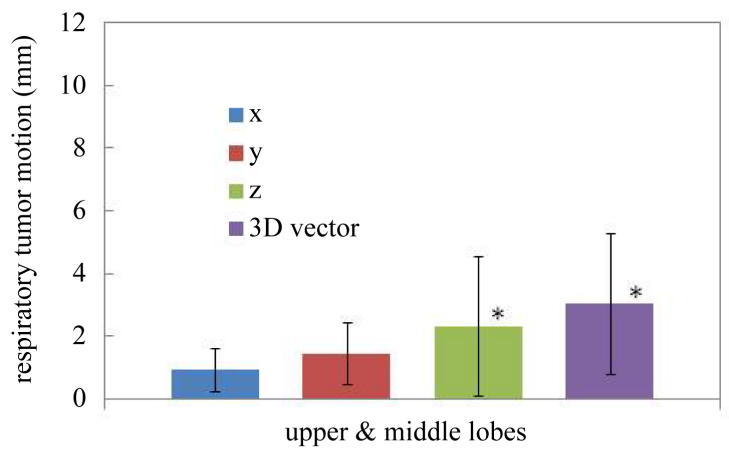

(a)

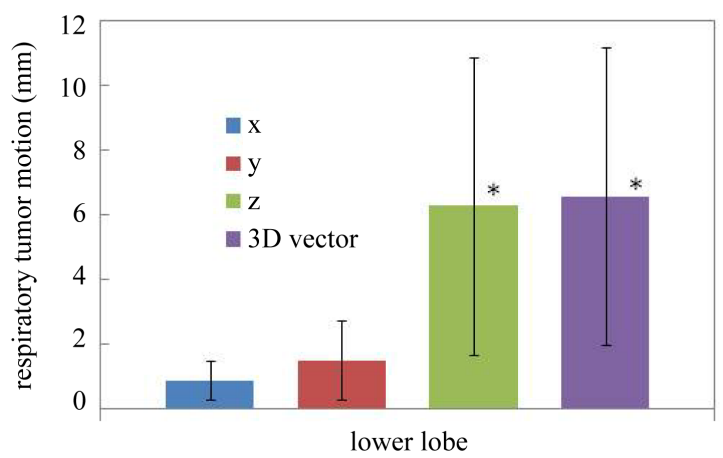

(b)

Figure 7. Respiratory tumor motion of $X$ (left-right), $Y$ (anterior-posterior), $\mathrm{Z}$ (caudal-cranial) and $3 \mathrm{D}$ vector $\left(\sqrt{\mathrm{X}^{2}+\mathrm{Y}^{2}+\mathrm{Z}^{2}}\right)$ for the (a) upper/middle and (b) lower lobes with the Air-Bag system $^{\mathrm{TM}}$, respectively. Box plots represent average tumor motion. Error bars represent one standard deviation. Statistical significance was evaluated using the Student unpaired t-test. *Significant difference between upper/middle and lower lobes ( $\mathrm{p}<$ $0.05)$.

one patient, with a lower lobe tumor. This decrease in organ motion is achieved by limiting diaphragmatic movement and reducing breathing volume. Respiratory tumor motion is generally $2 \mathrm{~cm}$, and is larger in the craniocaudal direction, where maximum motion of $3-4 \mathrm{~cm}$ is seen when the tumor is located just above the diaphragm [1].

Several solutions to account for respiratory tumor motion have been developed, including deep inspiration breath holding [11], the gating technique [8], and abdominal compression [9]. In deep inspiration breath holding, the therapist coaches the patient in achieving a reproducible deep inhalation breath hold during simulation and treatment. After holding the breath for a long period, however, the patient may tend to take deeper breaths, which may in turn affect the reproducibility of tumor position. In the gating technique, the therapist focuses on the gating system monitor and is prepared to intervene if the patient's breathing is very irregular or differs from that in simulation. These techniques result in a longer treatment time and the time and effort of additional staff. Forced shallow breathing uses abdominal compression, such as via the Stereotactic Body Frame system. Negoro et al. reported that abdominal pressure using the Stereotactic Body Frame reduced respiratory tumor motion significantly, from a range of 8 to $20 \mathrm{~mm}$ to a range of 2 to $11 \mathrm{~mm}$ [9]. Liu et al. reported that average tumor motion in the left-right, anterior-posterior and caudal-cranial directions was 1.2, 1.6, and 14.7 mm for lower lung tumors using an abdominal compression plate, respectively [12]. However, the stereotactic body frame system can obstruct the treatment beam arrangement, and beam attenuation caused by the body frame must be accounted for in the planning process [13]. In contrast, the Air-Bag System allows the beam to be arranged freely and seldom results in beam attenuation. Accordingly, it can also be used in radiotherapy for liver cancer. Ko et al. have reported a similar device, the "Air-injected blanket” (AIB), which provided a mean decrease in respira- 
tory tumor motion in the caudal-cranial and anterior-posterior directions from 2.6 to $0.7 \mathrm{~cm}$ and 2.7 to $1.3 \mathrm{~cm}$, respectively [14]. Application of pressure to the abdomen decreases respiratory tumor motion by both reducing diaphragm motion and limiting normal respiration. Given that it is placed between the entire surface of the patient's body and a HipFix ${ }^{\mathrm{TM}}$, we speculate that the Air-Bag System contributes to tumor position reproducibility primarily as an immobilization device.

Breathing control is critical to ensuring the accuracy of stereotactic irradiation in lung cancer patients. Breathing patterns can vary in magnitude, period and regularity during treatment sessions [15]. Onishi et al. developed the Abches (Apex Medical, Tokyo, Japan) system for respiration monitoring. This system reports respiratory tumor motion and enables respiration control with high accuracy and reproducibility, and thereby facilitates high-precision radiotherapy [16]. Visual or audiovisual biofeedback has been demonstrated to reduce breathing variation in mean cycle-to-cycle position, displacement, and period [17] [18]. The RPM system monitors the vertical displacement of two reflective markers attached to the patient's abdomen. Precise capture of the movement of the infrared reflective marker box using the infrared camera of RPM is difficult, however, as the results tend to be of relatively small amplitude and thus of limited quality. In our experience, placement of an infrared reflective plastic box on the abdomen as an external fiducial marker is inherently unstable. In contrast, combined use of the RPM and Air-Bag Systems allows the measurement of breathing patterns. This combination uses an exhaustive yet simplified evaluation of the three dimensional variation of movement of the chest and abdomen through respiration to provide a highly reliable motion signal, which greatly increases the reliability of the overall RPM system once the signal is fed to the infrared camera.

The most important muscle of inhalation is the diaphragm. Diaphragm motion likely has a close relationship with the tumor motion for lower lobe lung tumors, in which mechanical coupling between the tumor and diaphragm would be strongest. Eccles et al. reported that abdominal compression reduces three-dimensional liver tumor motion in most patients [19]. Abdominal compression with the Air-Bag System reduces movement of the diaphragm, which in turn decreases intrathoracic organ motion and leads to a reduction of the ITV, resulting in a decreased dose to the normal lung. Comparative studies with and without the Air-Bag System are needed to verify whether compression is also useful in reducing respiratory tumor motion.

\section{Conclusion}

The Air-Bag System is expected to provide both an effective reduction in the motion of lung tumors and a high quality, real-time breathing measurement signal. Our results support the clinical acceptability of treatment planning based on the Air-Bag System.

\section{References}

[1] Keall, P.J., Mageras, G.S., Balter, J.M., et al. (2006) The Management of Respiratory Motion in Radiation Oncology Report of AAPM Task Group 76. Medical Physics, 33, 3874-3900. http://dx.doi.org/10.1118/1.2349696

[2] Mori, S., Endo, M., Komatsu, S., Yashiro, T., Kandatsu, S. and Baba, M. (2007) Four-Dimensional Measurement of Lung Tumor Displacement Using 256 Multi Slice CT Scanner. Lung Cancer, 56, 56-67. http://dx.doi.org/10.1016/j.lungcan.2006.11.011

[3] Stevens, C.W., Munden, R.F., Forster, K.M., et al. (2001) Respiratory-Driven Lung Tumor Motion Is Independent of Tumor Size, Tumor Location, and Pulmonary Function. International Journal of Radiation Oncology, Biology, Physics, 51, 62-68.

[4] Chen, Q.S., Weinhous, M.S., Deibel, F.C., Ciezki, J.P. and Macklis, R.M. (2001) Fluoroscopic Study of Tumor Motion Due to Breathing: Facilitating Precise Radiation Therapy for Lung Cancer Patients. Medical Physics, 28, 1850-1856. http://dx.doi.org/10.1118/1.1398037

[5] Guckenberger, M., Meyer, J., Wilbert, J., et al. (2006) Cone-Beam CT Based Image-Guidance for Extracranial Stereotactic Radiotherapy of Intrapulmonary Tumors. Acta Oncologica, 45, 897-906. http://dx.doi.org/10.1080/02841860600904839

[6] ICRU (1993) Prescribing, Recording, and Reporting Photon Beam Therapy. ICRU Report Volume 50, International Commission on Radiation Units and Measurements, Bethesda.

[7] Seppenwoolde, Y., Lebesque, J.V., De Jaeger, K., et al. (2003) Comparing Different NTCP Models That Predict the Incidence of Radiation Pneumonitis. Normal Tissue Complication Probability. International Journal of Radiation Oncology, Biology, Physics, 55, 724-735. 
[8] Mageras, G.S. and Yorke, E. (2004) Deep Inspiration Breath Hold and Respiratory Gating Strategies for Reducing Organ Motion in Radiation Treatment. Seminars in Radiation Oncology, 14, 65-75. http://dx.doi.org/10.1053/j.semradonc.2003.10.009

[9] Negoro, Y., Nagata, Y., Aoki, T., et al. (2001) The Effectiveness of an Immobilization Device in Conformal Radiotherapy for Lung Tumor: Reduction of Respiratory Tumor Movement and Evaluation of the Daily Setup Accuracy. International Journal of Radiation Oncology, Biology, Physics, 50, 889-898.

[10] Miura, H., Masai, N., Oh, R.J., Shiomi, H., Sasaki, J. and Inoue, T. (2013) Approach to Dose Prescription of the Gross Tumor Volume for Lung Cancer with Respiratory Tumor Motion. Journal of Radiation Research, 54, 140-145. http://dx.doi.org/10.1093/jrr/rrs054

[11] Hanley, J., Debois, M.M., Mah, D., et al. (1999) Deep Inspiration Breath-Hold Technique for Lung Tumors: The Potential Value of Target Immobilization and Reduced Lung Density in Dose Escalation. International Journal of Radiation Oncology, Biology, Physics, 45, 603-611.

[12] Liu, H.H., Balter, P., Tutt, T., et al. (2007) Assessing Respiration-Induced Tumor Motion and Internal Target Volume Using Four-Dimensional Computed Tomography for Radiotherapy of Lung Cancer. International Journal of Radiation Oncology, Biology, Physics, 68, 531-540.

[13] Han, K., Cheung, P., Basran, P.S., Poon, I., Yeung, L. and Lochray, F. (2010) A Comparison of Two Immobilization Systems for Stereotactic Body Radiation Therapy of Lung Tumors. Radiotherapy \& Oncology, 95, 103-108. http://dx.doi.org/10.1016/j.radonc.2010.01.025

[14] Ko, Y.E., Suh, Y., Ahn, S.D., et al. (2005) Immobilization Effect of Air-Injected Blanket (AIB) for Abdomen Fixation. Medical Physics, 32, 3363-3366. http://dx.doi.org/10.1118/1.2047783

[15] Seppenwoolde, Y., Shirato, H., Kitamura, K., et al. (2002) Precise and Real-Time Measurement of 3D Tumor Motion in Lung Due to Breathing and Heartbeat, Measured during Radiotherapy. International Journal of Radiation Oncology, Biology, Physics, 53, 822-834.

[16] Onishi, H., Kawakami, H., Marino, K., et al. (2010) A Simple Respiratory Indicator for Irradiation during Voluntary Breath Holding: A One-Touch Device without Electronic Materials. Radiology, 255, 917-923. http://dx.doi.org/10.1148/radiol.10090890

[17] George, R., Ramakrishnan, V., Siebers, J.V., Chung, T.D. and Keall, P.J. (2006) Investigation of Patient, Tumour and Treatment Variables Affecting Residual Motion for Respiratory-Gated Radiotherapy. Physics \& Medicine in Biology, 51, 5305-5319. http://dx.doi.org/10.1088/0031-9155/51/20/015

[18] George, R., Chung, T.D., Vedam, S.S., et al. (2006) Audio-Visual Biofeedback for Respiratory-Gated Radiotherapy: Impact of Audio Instruction and Audio-Visual Biofeedback on Respiratory-Gated Radiotherapy. International Journal of Radiation Oncology, Biology, Physics, 65, 924-933.

[19] Eccles, C.L., Patel, R., Simeonov, A.K., Lockwood, G., Haider, M. and Dawson, L.A. (2011) Comparison of Liver Tumor Motion with and without Abdominal Compression Using Cine-Magnetic Resonance Imaging. International Journal of Radiation Oncology, Biology, Physics, 79, 602-608. 